\title{
ENFERMAGEM EM UNIDADE DE TERAPIA INTENSIVA NEONATAL: PERFIL DA PRODUÇÃO CIENTÍFICA BRASILEIRA
}

\author{
Aline Tomaz de Carvalho Teixeira Cruz ${ }^{1}$, Regina Cláudia Melo Dodt², Mônica Oliveira Batista Oriá ${ }^{3}$, Maria Dalva
} Santos Alves ${ }^{4}$

RESUMO: Trata-se de estudo descritivo do tipo bibliográfico que teve por objetivo caracterizar a produção científica brasileira de Enfermagem sobre Neonatologia e Recém-nascidos de risco no período entre 2004 e 2007 . A partir dos critérios adotados foram selecionados 31 artigos, os quais constituíram a amostra do estudo. Os resultados evidenciaram diversidade temática, de tipos de estudo e de abordagem; destacando-se o tema Assistência de Enfermagem, o método descritivo e a abordagem qualitativa. Os sujeitos mais frequentemente abordados foram os profissionais de equipes de saúde e os enfermeiros. A maioria dos autores apresentou propostas para a solução de problemas identificados, contudo, ainda são necessários mais estudos sobre a temática.

PALAVRAS-CHAVE: Enfermagem neonatal; Recém-nascido; Unidades de terapia intensiva neonatal

\section{NURSING IN A NEONATAL INTENSIVE THERAPY UNIT: A PROFILE OF BRAZILIAN SCIENTIFIC LITERATURE PRODUCTION}

\begin{abstract}
A descriptive, bibliographic study which aimed to characterize Brazilian nursing literature production concerning Neonatology and at-risk new-borns in the period from 2004 to 2007. Starting from the criteria adopted, 31 articles were selected, constituting the study's sample. The results showed evidence of thematic diversity, in types of study and approach. The theme of Nursing Assistance, the descriptive method and the qualitative approach stood out. The most frequently treated subjects were health team professionals and nurses. The majority of authors presented solutions for particular problems. Nevertheless, further studies on the theme are needed.
\end{abstract}

KEYWORDS: Neonatal nursing; New-born; Neonatal intensive therapy units.

\section{ENFERMERÍA EN UNIDAD DE TERAPIA INTENSIVA NEONATAL: PERFIL DE LA PRODUCCIÓN CIENTÍFICA BRASILEÑA}

RESUMEN: Se trata de estudio descriptivo del tipo bibliográfico que tuvo por objetivo caracterizar la producción científica brasileña de Enfermería sobre Neonatología y Recién nacidos de riesgo en el periodo entre 2004 y 2007. Considerándose los criterios adoptados, fueron seleccionados 31 artículos, los cuales constituyeron la muestra del estudio. Los resultados revelaron diversidad temática, de tipos de estudio y de abordaje; destacándose el tema Asistencia de Enfermería, el método fue el descriptivo y abordaje el cualitativo. Los sujetos más frecuentemente abordados fueron los profesionales de equipos de salud y los enfermeros. La mayoría de los autores presentó propuestas para la solución de problemas identificados, pero todavía son necesarios más estudios sobre la temática.

PALABRAS CLAVE: Enfermería neonatal; Recien nacido; Unidades de terapia intensiva neonatal.

${ }^{1}$ Enfermeira. Professora Substituta do Departamento de Enfermagem da Universidade Federal do Ceará-UFC.

${ }^{2}$ Enfermeira. Doutoranda no Programa de Pós-Graduação em Enfermagem da UFC.

${ }^{3}$ Enfermeira. Doutora em Enfermagem. Professora do Departamento de Enfermagem da UFC.

${ }^{4}$ Enfermeira. Psicóloga. Doutora em Enfermagem. Professora do Departamento de Enfermagem e do Programa de Pós-Graduação em Enfermagem da UFC.

\author{
Autor correspondente: \\ Regina Cláudia Melo Dodt \\ Universidade Federal do Ceará \\ Av. Heróis do Acre, 50 - 60743-760 - Fortaleza-CE-Brasil \\ E-mail: reginadodt@yahoo.com.br
}

Recebido: $14 / 04 / 10$

Aprovado: 29/09/10 


\section{INTRODUÇÃO}

Nas últimas décadas, a ciência e a tecnologia vêm avançando em todas as áreas, sobremaneira no campo da saúde, com destaque para a Neonatologia, que tem progredido com o surgimento de novos equipamentos, terapêuticas e conhecimentos. Em decorrência disto, observa-se o reflexo em Recém-Nascidos Prematuros (RNPT) e de baixo peso que, embora anteriormente fossem considerados inviáveis e atualmente sejam cada vez mais extremos, conseguem sobreviver.

Nascem, anualmente, no muneo vinte milhões de crianças prematuras e com baixo peso e, destas, um terço não chega a completar um ano de vida. A literatura nacional evidencia que o recém-nascido prétermo ( $<37$ semanas de gestação) e/ou recém-nascido de muito baixo peso $(<1500 \mathrm{~g})$ são os mais atingidos pela morbimortalidade no período neonatal ${ }^{(1)}$.

No Brasil, a Taxa de Mortalidade Infantil em 2007 foi de 19,3/1000 nascidos vivos, e a Taxa de Mortalidade Neonatal corresponde de $60 \%$ a $70 \%$ desta taxa em todas as regiões do país. As afecções perinatais representam $60 \%$ destas, destacando-se a prematuridade e suas complicações, sendo, portanto, uma das principais preocupações em saúde pública no país( ${ }^{(2)}$.

No Ceará, a melhoria na assistência à saúde, como no acompanhamento pré-natal, campanhas de vacinação e melhorias nas Unidades de Terapia Intensiva Neonatal (UTIN), acarretou em redução da mortalidade infantil em aproximadamente 50\%. No Município de Fortaleza/CE há forte contribuição da assistência aos recém-nascidos de baixo peso para a redução da taxa de mortalidade infantil. Contudo, esta taxa ainda se encontra elevada e a assistência terciária aos recémnascidos (RN) de risco permanece deficiente ${ }^{(3)}$.

Dados da literatura nacional mostram que esta taxa de morbimortalidade é de etiologia multifatorial, sofrendo fortes influências dos fatores socioeconômicodemográficos, políticos e biológicos, além do tipo de assistência à saúde ofertada, estando mais evidente em países em desenvolvimento que nos países desenvolvidos. Trata-se, portanto, de uma taxa de difícil controle, permanecendo estável ${ }^{(4)}$.

Contudo, a tecnologia e seus avanços possibilitaram que dentro das UTIN sejam oferecidos tratamentos mais eficazes, que ajudam a manter a vida dos recém-nascidos, prolongando-a e, assim, contribuindo para a diminuição da taxa de mortalidade infantil.

Em se tratando de UTIN, estas se encontram atualmente sobrecarregadas e marcadas pela tecnologia.
Assim, os profissionais precisam organizar seu espaço e suas tarefas entre os bebês e os equipamentos ${ }^{(1)}$. Daí ter-se a necessidade de uma equipe multidisciplinar, especializada, capaz de prestar atendimento individualizado ao bebê e à família.

Com o advento das incubadoras, do uso de medicamentos como o surfactante e com o surgimento de novos antimicrobianos, além da terapia com oxigênio, alcançou-se uma maior sobrevida dos bebês em estados mais graves e de menor peso, que acabam permanecendo por mais tempo sob tratamento intensivo.

Contudo, o ambiente das UTIN é bastante distinto do intrauterino, com excesso de luminosidade, ruídos e procedimentos invasivos. Além disso, quanto mais prematuro é o RN, maior a quantidade de manipulações a que é submetido. Tudo isto pode acarretar em sequelas, tanto físicas como do sistema neuromotor, com grande risco de desenvolver distúrbios comportamentais relacionados ao estresse. Assim, o ambiente da UTIN pode contribuir significativamente para essas alterações, que podem persistir por toda a vida e se tornar incapacitantes ${ }^{(5)}$.

Voltando os olhares à Enfermagem Neonatal, trata-se de uma profissão indispensável no quadro de recursos humanos de uma UTIN. Seus agentes atuam nos cuidados clínicos curativos e preventivos. O enfermeiro deve aliar seus conhecimentos, técnicas e experiências à sensibilidade e ao relacionamento interpessoal terapêutico ${ }^{(6)}$, com o objetivo de fornecer um cuidado seguro a estes clientes. Frequentemente se encontram clinicamente instáveis, e suas famílias estão ansiosas e inseguras diante de tamanha fragilidade e da possibilidade de perda iminente.

$O$ cuidado de enfermagem enquanto conhecimento/saber específico transcende um simples ato simbólico e/ou técnica prescritiva ${ }^{(7)}$. Trata-se de um processo social, institucional, profissional e pessoal dinâmico e gradual que exige tempo, atitude, comportamento e um esforço incansável dos profissionais em buscar novas formas de ação/interação ${ }^{(8)}$.

Atualmente, sabe-se que o enfermeiro tem um importante papel na manutenção das condições de vitalidade dos prematuros, devendo fundamentar suas ações em conhecimentos científicos ${ }^{(9)}$. Esta busca pelo conhecimento deve atender também às exigências do mercado de trabalho, que se encontra saturado, exigindo cada vez maior titulação dos profissionais.

Hoje, o perfil dos profissionais, principalmente do enfermeiro, deve contemplar uma formação generalista, humanista, crítica e reflexiva ${ }^{(10)}$. Esse 
profissional deve ser capaz de atuar com ética, senso de responsabilidade e compromisso com a cidadania.

A UTIN apresenta-se como um espaço de trabalho que exige maiores conhecimentos e habilidades do profissional de enfermagem. Pode tornar-se, ainda, um campo de pesquisa para aprimoramento de suas práticas.

A importância da utilização do espaço de trabalho do enfermeiro para a realização de pesquisas é reconhecida como essencial, mas ainda há entraves para seu desenvolvimento ${ }^{(11)}$. A ação investigativa em campo clínico possibilita que o enfermeiro adquira, produza e aprofunde conhecimentos, atualize e avalie suas práticas, cresça profissionalmente pelo estímulo à reflexão de novas formas de conduzir seu trabalho e desperte para a necessidade do conhecimento estruturado de forma científica ${ }^{(12)}$.

Sobre as questões do cotidiano na Enfermagem, este é um dos espaços que, por excelência, precisa ser utilizado não apenas para o uso e a socialização do conhecimento já posto, mas também para a sua (re) construção. Este processo deve ser potencializado pelo posicionamento crítico diante do conhecimento produzido, pela criatividade e pelo improviso, que têm se tornado preponderantes dentro desta construção do saber $^{(12)}$.

O presente trabalho teve como objetivo principal caracterizar o perfil da produção científica brasileira de Enfermagem sobre Neonatologia e RecémNascidos de risco, no período de 2004 a 2007, por meio da realização de levantamento bibliográfico nas principais revistas brasileiras de Enfermagem.

\section{METODOLOGIA}

Trata-se de um estudo de caráter descritivo, pois objetiva tratar das características do objeto em estudo. É também bibliográfico e documental, em que foram utilizadas fontes primárias e secundárias para a sua construção. Foram realizadas buscas de artigos que tratassem sobre Neonatologia e Enfermagem, presentes em seis periódicos brasileiros de enfermagem disponíveis no Scientific Electronic Library Online (SciELO) e na Base de Dados de Enfermagem (BDENF).

Foram selecionadas aleatoriamente seis revistas para a pesquisa: Texto \& Contexto - Enfermagem; Acta Paulista de Enfermagem; Revista Latino Americana de Enfermagem; Revista da Escola de Enfermagem da USP; Revista Mineira de Enfermagem; e Revista Brasileira de Enfermagem.
No Banco de Dados do SciELO, ao abrir a página de cada revista disponível, foram utilizados os seguintes termos no campo para pesquisa: "enfermagem e neonatologia", "enfermagem e prematuro", "cuidado e terapia intensiva neonatal e enfermagem" e "enfermagem e neonatal". A partir destas associações, foram encontrados 64 artigos. O Banco de Dados de Enfermagem foi acessado por meio do site da BVS e usado o termo "enfermagem neonatal" no campo de pesquisa. A partir dos descritores citados anteriormente, foram encontrados 224 artigos, totalizando, portanto, 288 artigos, dos quais foram lidos os resumos, com o objetivo de delimitar a amostra.

Para compor o presente estudo, foram selecionados os artigos de Enfermagem de acordo com os seguintes critérios de inclusão: artigos publicados entre os anos de 2004 e 2007; artigos que estivessem disponíveis na íntegra; e artigos que tratassem de $\mathrm{RN}$ prematuros ou de baixo peso internados em UTIN, de suas famílias e dos profissionais que trabalham na UTI. Os critérios de exclusão foram os seguintes: artigos que tratavam de RN fora da situação de risco; RN com má formação congênita; $\mathrm{RN}$ internados por motivo de cirurgias; $\mathrm{RN}$ em alojamento conjunto ou enfermaria; artigos que tratavam da atenção ao recém-nascido em unidades básicas ou em Programa de Saúde da Família; artigos sobre os acadêmicos de enfermagem, ou de ensino sobre Enfermagem Neonatal.

Para cada texto selecionado, após sua leitura integral, foram preenchidas fichas com os seguintes itens para análise: 1) Ano de Publicação; 2) Nome da Revista; 3) Tema; 4) Abordagem TeóricoMetodológica; 5) População Estudada; 6) Análise dos Dados; 7) Apresentação de Propostas para a solução de problemas. Foram feitas as contagens de cada item e elaborados quadros para análise.

\section{RESULTADOS}

Foram encontrados 31 artigos que atenderam aos critérios adotados para inclusão. Quanto ao ano de publicação, foram encontrados 5 artigos no ano de 2004, 6 no ano de 2005, 16 no ano de 2006 e 4 no ano de 2007, perfazendo uma média de aproximadamente 8 artigos/ano. Foi em 2006 que ocorreram 51,6\% das publicações nesta área, apresentando-se com, aproximadamente, o dobro da média. 
Conforme o quadro 1, que apenas uma revista pertencia ao estrato mais elevado da área (A2), de acordo com a nova definição dos estratos do Qualis Periódicos da Coordenação de Aperfeiçoamento de pessoal de Nível Superior - CAPESP ${ }^{(13)}$. Três das revistas selecionadas apresentaram-se com estrato inferior, cuja circulação é em nível nacional e possuem indexação em pelo menos quatro bases de dados nacionais ou internacionais (B1), e duas com estrato mais inferior (B2), de circulação nacional e indexadas em pelo menos três bases de dados nacionais ou internacionais ${ }^{(13)}$.

Foi constatado que nenhuma das revistas apresentava-se dentro do estrato mais elevado, A1, de peso 100, já que, de acordo com a CAPES ${ }^{(13)}$, a Enfermagem é uma área "em consolidação", quando comparada com as demais áreas, sendo o estrato mais elevado (A1) pouco povoado por publicações da área.

Quanto ao número de artigos por revista (Quadro

Quadro 1 - Classificação dos Periódicos de Enfermagem, segundo os Estratos da CAPES. Fortaleza, 2008

\begin{tabular}{lcc}
\hline Periódicos de Enfermagem & Estrato & Peso \\
\hline $\begin{array}{l}\text { Revista Latino-Americana de } \\
\text { Enfermagem }\end{array}$ & A2 & 85 \\
$\begin{array}{l}\text { Revista da Escola de Enfermagem } \\
\text { da USP }\end{array}$ & B1 & 70 \\
Texto \& Contexto - Enfermagem & B2 & 50 \\
Acta Paulista de Enfermagem & B1 & 70 \\
Revista Mineira de Enfermagem & B2 & 50 \\
Revista Brasileira de Enfermagem & B1 & 70 \\
\hline
\end{tabular}

Fonte: CAPES

2), a maior parte das publicações (32,2\%) encontra-se em revista de ampla circulação e indexados em base de dados nacional e internacional; ou seja, pertencente ao estrato mais elevado das selecionadas (A2).

A Revista Latino-Americana de Enfermagem apresentou maior número de publicações, totalizando 10 artigos $(32,2 \%)$, o que a coloca acima da média de aproximadamente 5 artigos/revista (5,2 artigos/ revista). Sabe-se que este periódico pertence à Escola de Enfermagem de Ribeirão Preto, da Universidade de São Paulo, e ao centro colaborador da Organização Pan-Americana da Saúde/Organização Mundial da Saúde para o Desenvolvimento da Pesquisa em Enfermagem.

Em relação aos estudos abordados pelos pesqui-
Quadro 2 - Distribuição do número de artigos por revista. Fortaleza, 2008

\begin{tabular}{lcc}
\hline Periódicos de Enfermagem & N & \% \\
\hline $\begin{array}{l}\text { Revista Latino-Americana de } \\
\text { Enfermagem }\end{array}$ & 10 & 32,2 \\
$\begin{array}{l}\text { Revista da Escola de Enfermagem da } \\
\text { USP }\end{array}$ & 03 & 9,7 \\
Texto \& Contexto - Enfermagem & 05 & 16,1 \\
Acta Paulista de Enfermagem & 06 & 19,3 \\
Revista Mineira de Enfermagem & 03 & 9,7 \\
Revista Brasileira de Enfermagem & 04 & 13,0 \\
\hline TOTAL & $\mathbf{3 1}$ & $\mathbf{1 0 0}$ \\
\hline
\end{tabular}

Fontes: SciELO e BDENF

sadores, foram encontradas seis diferentes temáticas (Quadro 3), sendo o Processo e Assistência de Enfermagem a mais abordada, totalizando $11(35,5 \%)$ publicações.

Quadro 3 - Distribuição do número de artigos em revistas de Enfermagem por tema abordado. Fortaleza, 2008

Quanto aos tipos de estudo, o estudo descritivo

\begin{tabular}{lcc}
\hline Temas Abordados & N & \% \\
\hline Processo e Assistência de Enfermagem & 11 & 35,5 \\
Relação Pais/RN/Equipe de Saúde & 06 & 19,3 \\
Método Canguru & 05 & 16,1 \\
Profissionais de Enfermagem & 04 & 13,0 \\
Dor & 03 & 9,7 \\
Ruído & 02 & 6,4 \\
\hline TOTAL & $\mathbf{3 1}$ & $\mathbf{1 0 0}$ \\
\hline
\end{tabular}

Fontes: SciELO e BDENF

apresentou predominância, representando mais da metade dos estudos produzidos, com 21 (67,7\%) artigos. Em segundo lugar, apresentou-se o estudo de revisão bibliográfica, com seis (19,4\%) artigos. Em seguida, vieram três $(9,7 \%)$ estudos de caso e um $(3,2 \%)$ estudo experimental.

De acordo com a abordagem teórico-metodológica adotada pelos autores, 23 artigos utilizaram a abordagem qualitativa, perfazendo $74,2 \%$ das publicações. A abordagem quantitativa foi utilizada em seis $(19,4 \%)$ artigos e a qualitativa/quantitativa em dois $(6,4 \%)$. Da amostra, 24 (77,4\%) artigos representaram investigações envolvendo seres humanos, dentre eles três 
com abordagem quantitativa, dois com abordagem qualitativa/quantitativa e 19 de natureza qualitativa. Apenas 7 (22,6\%) artigos não realizaram pesquisas com seres humanos, representadas por medidas em aparelhos (um artigo) ou artigos de revisão bibliográfica (seis artigos).

Os sujeitos envolvidos foram somente Enfermeiros, Equipes de Saúde, Mães dos Neonatos, Equipe de Enfermagem (enfermeiros, técnicos e auxiliares de enfermagem), Mães e Equipes de Enfermagem e Pais dos Recém-Nascidos (Quadro 4).

Quadro 4 - Distribuição do número de artigos quanto aos sujeitos das pesquisas. Fortaleza, 2008

\begin{tabular}{lcc}
\hline Sujeitos das Pesquisas & $\mathbf{N}^{*}$ & $\mathbf{\%}$ \\
\hline Enfermeiros & 05 & 20,8 \\
Equipes de Saúde & 05 & 20,8 \\
Mães & 04 & 16,7 \\
Equipes de Enfermagem & 04 & 16,7 \\
Mães e Equipe & 02 & 8,3 \\
Recém-Nascido & 02 & 8,3 \\
Pais & 01 & 4,2 \\
Pais e Recém-Nascidos & 01 & 4,2 \\
\hline TOTAL & $\mathbf{2 4}$ & $\mathbf{1 0 0}$ \\
\hline
\end{tabular}

Fontes: Scielo e BDENF

*Artigos que realizaram estudos com seres humanos. Não foram incluídos, portanto, as revisões bibliográficas ou estudos com equipamentos

Foi verificado que $24(77,4 \%)$ artigos apresentaram propostas na tentativa de melhorias e apenas $7(22,6 \%)$ não deram nenhuma sugestão.

\section{DISCUSSÃO}

De acordo com a CAPES ${ }^{(13)}$, Qualis é uma lista de veículos que divulga a produção intelectual de pós-graduação Stricto sensu (Mestrado e Doutorado), a partir da qual se obtém o respaldo científico do periódico.

Tradicionalmente, os descobrimentos científicos ganham reconhecimento e credibilidade em função de sua publicação em revistas científicas de prestígio. Assim, justifica-se a busca da Enfermagem Neonatal em produzir, cientificamente, artigos de qualidade. Contudo, estudo refere que ainda não existem produções científicas de Enfermagem da América Latina indexadas no Institute for Scientific Information, que oferece cobertura às pesquisas mais importantes no mundo, tendo, portanto, pouca visibilidade internacional $^{(14)}$.

A Universidade de São Paulo é considerada a melhor universidade latinoamericana e uma das 120 melhores do mundo, de acordo com a edição de 2007 do Academic Ranking of World Universities, o que justifica seu maior número de publicações. Esta disponibilizou mais publicações por meio da Revista da Escola de Enfermagem da USP, de Ribeirão Preto, classificada de acordo com a CAPES ${ }^{(13)}$ como "B1"; porém, este periódico apresentou apenas três publicações na área de interesse do presente estudo.

O processo de enfermagem é uma abordagem para a solução de problemas, visando satisfazer as necessidades de enfermagem e de cuidados de saúde de uma pessoa. É por meio dele que se direciona a assistência do profissional enfermeiro e de sua equipe, quando a teoria é vinculada à prática. Estes temas continuam a constituir objeto de preocupação de enfermeiros em diferentes âmbitos de atuação, sejam de ensino, pesquisa e assistência ${ }^{(15)}$. O processo de enfermagem fornece estrutura para a tomada de decisão durante a assistência de enfermagem, tornando-a mais científica e menos intuitiva.

Os profissionais de enfermagem almejam a cientificidade de sua profissão e esta só será alcançada com a utilização de instrumentos científicos que subsidiem a prática profissional ${ }^{(16)}$. Eles buscam, portanto, reconhecimento profissional conjugado à autonomia, o que permite a definição de desafios e objetivos, bem como a forma como estes profissionais se relacionam e se apresentam para a equipe de saúde e para a sociedade em geral ${ }^{(17)}$.

Acreditamos que a autonomia na profissão só será adquirida quando toda a classe começar a utilizar essa metodologia científica em suas ações, ou seja, quando estiver em prática a aplicação sistemática do processo de enfermagem ${ }^{(18)}$.

Apesar da tendência ao estudo descritivo nos trabalhos consultados, foi constatada a diversidade de métodos utilizados para a sua realização. Vivemos em uma época de transição paradigmática, na qual emergem diferentes formas de pensar o mundo e as ciências ${ }^{(19)}$. Ainda mais, o enfermeiro no mundo globalizado deve saber articular seus conhecimentos, sendo não só experiente em práticas, mas também nas ciências que lhes fundamentam ${ }^{(19)}$.

A metodologia qualitativa de pesquisa dispõe de 
instrumentos adaptáveis ao processo de cuidar em enfermagem, pois possui interfaces comuns relativas ao paradigma e às perspectivas filosóficas, sociais e culturais de se vislumbrar os fenômenos ${ }^{(20)}$. Além disso, esta metodologia fornece subsídios para reorientar as práticas profissionais ao complementar e conduzir para a construção e consolidação do conhecimento, na medida em que apontam evidências científicas para nortear a assistência ${ }^{(20)}$. Percebe-se uma tendência dos pesquisadores em Enfermagem pela pesquisa qualitativa, caracterizada pela preocupação com a compreensão e a interpretação do fenômeno, considerando o significado que os outros dão às suas práticas.

Diante da norma que regulamenta a pesquisa com seres humanos, todos os autores dos artigos afirmam ter respeitado os aspectos éticos, explicitando em seus estudos que utilizaram Termos de Consentimento e que foram submetidos e aprovados por Comitês de Ética em Pesquisa (CEP).

A busca de conhecimento utilizando diversos sujeitos da pesquisa demonstra o interesse em conhecer a Enfermagem Neonatal sob diversas óticas. O conhecimento científico interfere nas relações da enfermagem com a equipe de saúde, com a clientela e com a instituição à qual pertence ${ }^{(18)}$. À medida que o ser humano busca saber sobre si mesmo e sobre seu meio, aumentam as possibilidades de intervir sobre o âmbito individual, o coletivo e o planetário(21).

As propostas para a solução de problemas foram consideradas quando os autores indicavam pontos pontos considerados negativos e sugeriam estratégias para a mudança desses quadros. Verifica-se, assim, a presença de reflexões críticas sobre suas temáticas, além da preocupação de grande parte dos pesquisadores de Enfermagem em modificar as situações atuais, em busca de melhorias.

A detecção de problemas e criação de diretrizes para solucioná-los ou preveni-los são características do profissional crítico e do pesquisador, que devem procurar a verdade e o saber por meio da honestidade intelectual, do desinteresse pessoal, da defesa à verdade e da crítica à falsidade ${ }^{(21)}$. Apontar problemas existentes pode, também, abrir caminhos para novos estudos, além de fornecer meios para a melhoria na assistência e no próprio método da pesquisa.

Apesar de ainda existir sobre a Enfermagem a influência do modelo clínico, em que o objeto de trabalho é o corpo e a doença, a profissão vem buscando encontrar maneiras para desenvolver um corpo de conhecimentos próprios ${ }^{(12)}$. Assim, identifica-se a tentativa de reconhecimento profissional, autonomia e legítima busca de identidade.

\section{CONCLUSÃO}

De acordo com os dados, foi possível constatar a versatilidade da produção de Enfermagem sobre a assistência aos RN de risco internados em UTIN.

No entanto, reconhecemos a limitação do estudo, que ficou restrito ao idioma português e aos periódicos nacionais.

A produção sobre a Enfermagem e seus cuidados aos RN internados em UTIN ainda é deficiente, não em temáticas, mas em quantidade de estudos. Faz-se necessário maior número de publicações sobre os RN, interpretação de seus sinais/sintomas e iatrogenias, visando um cuidado ético e a minimização das seqüelas decorrentes de possíveis negligências.

Visto que a produção de conhecimento deve acompanhar as mudanças e tendências, e que o pesquisador de Enfermagem deve buscar a criatividade, não se deve encerrar as buscas de novos conhecimentos nesta temática.

\section{REFERENCIAS}

1. Ministério da Saúde (BR). Secretaria de Políticas de Saúde. Área de Saúde da Criança. Atenção humanizada ao recém-nascido de baixo peso. Método Mãe-Canguru: manual do curso. Brasília: Ministério da Saúde; 2002.

2. Brasil. Ministério da Saúde. Secretaria de Atenção a Saúde. Manual dos Comitês de Prevenção do Óbito Infantil e Fetal. Brasília: Ministério da Saúde; 2009.

3. Castro ECM, Leite AJM. Hospital mortality rates of infants with birth weight less than or equal to $1,500 \mathrm{~g}$ in the northeast of Brazil. J Pediatr. 2007;83(2):27-32.

4. Araujo BF, Tanaka ACA, Madi JM, Zatti H. Estudo da mortalidade de recém-nascidos internados na UTI neonatal do Hospital Geral de Caxias do Sul, Rio Grande do Sul. Rev Bras Saúde Mater Infant. 2005;5(4):463-69.

5. Alves AMA, Santos IMM, Porto F, Figueiredo NMA. Cuidados para o recém-nascido enfermo. In: Figueiredo NMA, organizadora. Ensinando a cuidar da mulher, do homem e do recém-nascido. $4^{\mathrm{a}}$ ed. São Paulo: Difusão enfermagem, 2003. 
6. Gaiva MAM. O cuidar em unidades de cuidados intensivos neonatais: em busca de um cuidado ético e humanizado. Cogitare Enferm. 2006;11(1):61-6.

7. Backes DS, Sousa FGM, Mello ALSF, Erdmann AL, Nascimento KC, Lessmann JC. Concepções de cuidado: uma análise das teses apresentadas para um programa de pós-graduação em enfermagem. Texto \& Contexto Enferm. 2006;15(n. esp):71-8.

8. Erdmann AL, Sousa FGM, Backes DS, Mello ALSF. Construindo um modelo de sistema de cuidados. Acta Paul Enferm. 2007;20(2):180-85.

9. Scochi CGS, Riul MJS, Garcia CFD, Barradas LS, Pileggi SO. Cuidado individualizado ao pequeno prematuro: o ambiente sensorial em unidade de terapia intensiva neonatal. Acta Paul Enferm. 2001;14(1):0916.

10. Pai DD, Schrank G, Pedro ENR. O enfermeiro como ser sócio-político: refletindo a visibilidade da profissão do cuidado. Acta Paul Enferm. 2006; 19(1): 82-87.

11. Dyniewicz AM, Rivero GMG. Metodologia da pesquisa para enfermeiras de um hospital universitário. Rev Latino-Am Enfermagem. 2005;13(3):354-63.

12. Lucena AF, Paskulin LMG, Souza MF, Gutiérrez MGR. Construção do conhecimento e do fazer enfermagem e os modelos assistenciais. Rev Esc Enferm USP. 2006;40(2):292-8.

13. Ministério da Educação (BR). Coordenação de Aperfeiçoamento de Pessoal de Nível Superior (CAPES) [Internet]. Brasília: Coordenação de Aperfeiçoamento de Pessoal de Nível Superior (CAPES); [acesso em 30 jan 2009]. Qualis CAPES; [1 tela]. Disponível: http://www.capes.gov.br.

14. Marziale MHP. Produção científica da enfermagem brasileira: a busca pelo impacto internacional. Rev Latino-Am Enfermagem. 2005;13(3):285-6.

15. Figueiredo RM, Zem-Mascarenhas SH, Napoleão AA, Camargo AB. Caracterização da produção do conhecimento sobre sistematização da assistência de enfermagem no Brasil. Rev Esc Enferm USP. 2006: 40(2):299-303.

16. Carraro TE, Kletemberg DF, Gonçalves LM. O ensino da metodologia da assistência de enfermagem no Paraná. Rev Bras Enferm. 2003;56(5):499-501.

17. Gomes AMT, Oliveira DC. Estudo da estrutura da representação social da autonomia profissional em enfermagem. Rev Esc Enferm USP. 2005;39(2):145-53.

18. Tannure MC, Gonçalves AMP, Carvalho DV. O processo de enfermagem In: Tannure MC, Gonçalves AMP. Sistematização da assistência de enfermagem: guia prático. Rio de Janeiro: Guanabara Koogan; 2009.

19. Ferreira HM. A totalidade do conhecimento da enfermagem: uma abordagem curricular. Acta Paul Enferm. 2003;16(1): 56-65.

20. Merighi MAB, Kimura AF. Enfocando estratégias de ensino de pesquisa qualitativa utilizadas no curso de graduação da Escola de Enfermagem da Universidade de São Paulo. REME - Rev Min Enferm. 2004:8(3):398-401.

21. Padilha MICS, Ramos FRS, Borenstein MS, Martins CR. A responsabilidade do pesquisador ou sobre o que dizemos acerca da ética em pesquisa. Texto \& Contexto Enferm. 2005;14(1):96-105. 